\title{
Contribution des essais aux activités de la SNCF en matière d'amélioration permanente de la sécurité et de la préservation de l'environnement
}

\author{
Francis Cocheteux ${ }^{a}$, Christian Proux et Jean-Jacques Viet \\ Agence d'Essai Ferroviaire, 21 avenue Salvador Allende, 94407 Vitry-sur-Seine, France
}

Reçu le 10 avril 2002, accepté le 5 décembre 2003

\begin{abstract}
Résumé - Sécurité - Performance - Endurance - Fiabilité, sont les mots clés du vocabulaire des concepteurs et des personnels de maintenance des matériels ferroviaires. La préservation de l'environnement s'inscrit comme un nouvel élément à ces préoccupations dans le cadre du développement durable. Bien que les performances des moyens de simulations numériques ne cessent de progresser, la SNCF continue de s'appuyer sur l'expérimentation pour vérifier et valider les dispositions prises par les bureaux d'étude. L'Agence d'Essai Ferroviaire, à travers les compétences de son personnel et de ses moyens d'essais, contribue activement à la maîtrise de ces objectifs.
\end{abstract}

Mots clés : Sécurité / essais / chemin de fer / développement durable

\begin{abstract}
SNCF tests contribution concerning permanent improvement of security and environment preservation. Safety - Performance - Endurance - Reliability, are the keywords of the vocabulary of the engineers and the staffs of rolling stock maintenance. The preservation of the environment appears as a new element to these preoccupations in accordance with sustained development. Although the performances of the numeric simulations do not stop progressing, SNCF keep on to lean on experimentation to verify and to validate the arrangements taken by the design department. The French Railway Centre, through knowledge of its staff and its testing, equipment actively contributes to reach these objectives.
\end{abstract}

Key words: Safety / test / railway / sustained development

\section{Introduction}

La SNCF se doit d'assurer à ses clients, à son personnel et à la collectivité, le haut niveau de sécurité qu'ils attendent et la mise en œuvre de toutes les dispositions nécessaires à la protection de l'environnement. C'est aussi des enjeux déterminants pour la pérennité et le développement du mode ferroviaire.

Pour répondre à ces attentes, l'entreprise s'organise par la maîtrise des processus de conception et de production concernant la sécurité et le respect de l'environnement :

- en tant que transporteur [1], dans l'exécution de ses activités commerciales et de ses missions de service public qui lui sont confiées par l'État [2] ou les collectivités territoriales,

- en tant que gestionnaire d'infrastructure pour le compte de RFF.

a Auteur correspondant : francis.cocheteux@sncf.fr
Le système ferroviaire est un système complexe reposant essentiellement sur quatre composants qui nécessitent que l'on veille en permanence à leur cohérence et à leur bonne intégration tout au long de sa conception, de son évolution et, plus généralement, tout au long de son cycle de vie :

- L'homme

C'est l'homme qui définit, conçoit, met en œuvre et maintient les différents éléments du système. C'est également lui qui définit les organisations au sein desquelles s'inscrivent ses actions.

- Les équipements

Par équipements, on entend les constituants de l'infrastructure et les constituants du matériel roulant, ainsi que les moyens de guidage et de communication entre le sol et le mobile.

- Les procédures

Il s'agit de l'ensemble des règles prescrites pour la définition, la conception, l'exploitation et la maintenance 
du système : règles légales, normes, modes opératoires, etc.

- L'environnement

L'environnement est constitué d'éléments matériels et humains (clients, tiers) formant le cadre de mise en œuvre de l'activité exercée et susceptibles d'influer sur la nature et le niveau des résultats.

\section{En matière de sécurité}

L'objectif de sécurité est clairement fixé par l'État [3] et est décliné au sein de l'entreprise [4].

\subsection{Les différentes approches de sécurité}

S'agissant de la définition des objectifs globaux de sécurité, il existe quatre approches possibles [5] : tifs

- L'approche interne au monde des transports collec-

Les objectifs de sécurité doivent être d'un ordre de grandeur comparable au performances de sécurité déjà observées dans le monde des transports collectifs (principe GAMAB - Globalement Au Moins Aussi Bon) afin d'assurer la sécurité des circulations.

- L'approche médiatique

Elle considère que le nombre d'accidents qui se sont produits ne constitue pas un résultat statistiquement significatif et il est à mettre en regard des probabilités d'apparition des grandes catastrophes naturelles imprévisibles et imparables.

- L'approche externe

Elle se fonde sur la notion de risque ambiant que vit quotidiennement chaque individu. Le transport constituant une partie de ce risque, tout nouveau service mis à la disposition de l'usager ne doit pas faire augmenter le risque ambiant (principe MEM - Mortalité Endogène Minimum).

- L'approche exploitant

Elle met en œuvre les lois de probabilité. Tout système possède une certaine probabilité de provoquer un accident. Le dimensionnement sera fait pour minimiser cette probabilité d'accident en intégrant le coût des améliorations (principe ALARP - As Low As Reasonably Practicable).

L'approche retenue par la SNCF est concrète, à savoir que tout système nouveau ou toute modification à un système en exploitation doit offrir un niveau global de sécurité au moins équivalent à celui des systèmes existants offrant des services comparables (principe GAME). Une telle approche a le mérite de maintenir une dynamique de progrès en minimisant le risque du «toujours plus ». De plus, cette approche est essentiellement déterministe car elle se base sur des référentiels existants sans pour autant rejeter l'approche probabiliste qui reste nécessaire dans le cas de sous-systèmes technologiques innovants.
Pour chaque nouveau projet (système nouveau ou modification), une approche système globale est appliquée avec toutes ses composantes :

- infrastructures,

- installations techniques et de sécurité,

- matériels roulants,

- opérateurs,

- procédures et règlements de sécurité,

- interfaces entre ces composantes.

Cette approche reste vraie pour chacune des phases de son cycle de vie :

- définition,

- conception et réalisation,

- essais et mise en service,

- exploitation et maintenance,

- sélection, formation, qualification, suivi et maintien des connaissances des opérateurs.

\subsection{L'importance du facteur humain}

L'homme est au cœur du système : il définit, conçoit, met en œuvre, contrôle et maintient.

Aucun système ne peut fonctionner de manière satisfaisante si la place et le rôle de l'homme n'ont pas été étudiés avec suffisamment de rigueur.

Mis au cœur d'un système bien conçu, l'homme joue un rôle positif important car grâce à ses facultés d'analyse et d'anticipation, il est capable de redresser des situations exceptionnelles, ce que les automatismes ne savent pas toujours faire.

À l'inverse, tout système de sécurité doit être conçu en tenant compte du fait que l'homme est faillible, en respectant le principe de redondance des sécurités.

De plus, le retour d'expérience (REX) est conçu et réalisé pour aider à détecter les points de faiblesse affectant la sécurité ferroviaire.

\subsection{L'implication des essais dans la sécurité des circulations}

L'Agence d'Essai Ferroviaire (AEF) de Vitry-surSeine, composante de la SNCF joue un rôle important dans cette approche système par les essais qu'elle met en oeuvre notamment sur les matériels roulants et leurs constituants tant du point de vue de la sécurité des circulations que du coût du cycle de vie. Son champ d'action s'étend de la conception initiale au recyclage des matériels en fin de vie (Fig. 1).

L'AEF est également sollicitée pour des projets innovants de la SNCF où la dimension technique doit être éprouvée et validée.

À titre d'exemple, lors de la construction de la nouvelle ligne à grande vitesse entre Valence et Marseille (LN5) qui traverse la vallée du Rhône, il a fallu intégrer l'incidence du Mistral pour garantir la sécurité des circulations des TGV, les rafales de vent pouvant atteindre plus de $180 \mathrm{~km} \mathrm{~h}^{-1}$. Pour cela, l'équation vents 


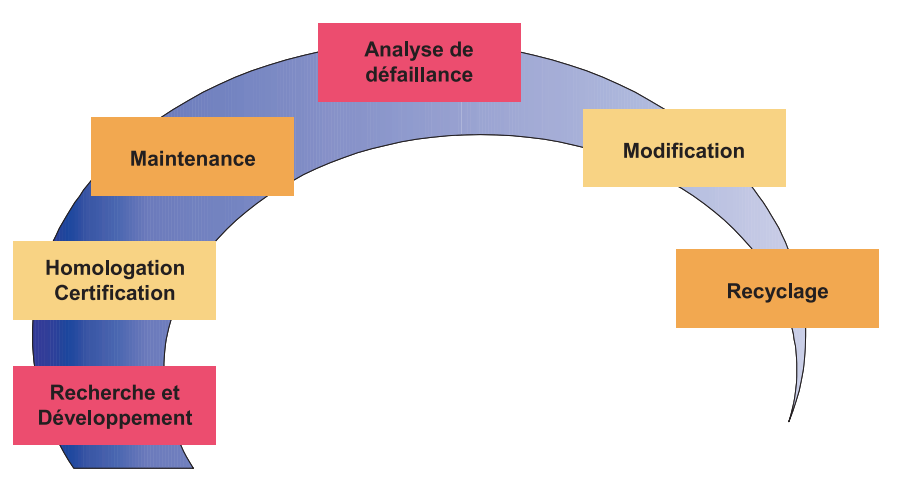

Fig. 1. Périmètre d'action de l'Agence d'Essai Ferroviaire.

traversiers-vitesse des circulations-sécurité des circulations a été décomposée en sept étapes :

- Analyse topographique de la ligne (viaduc, remblai, talus...).

- Étude météorologique des quatre dernières années.

- Étude en soufflerie du matériel roulant puis calcul d'abaques limites de circulation (Fig. 2).

- Positionnement des points météo sur les abaques pour déterminer la « criticité » des sites.

- Installation de protection physique sur les sites les plus critiques.

- Mise en place d'abaques complémentaires lors des périodes hivernales.

- Ajustement des abaques en fonction du délestage maximal autorisé, phase expérimentale.

Cette dernière étape consiste à mesurer simultanément, lors des circulations des TGV, la vitesse du vent, son orientation et les forces verticales exercées par chaque roue sur le rail afin de calculer le délestage dû au vent.

Les essais réalisés tant au sol qu'à bord de rames TGV instrumentées par l'AEF ont permis de valider les seuils de déclenchement des ralentissements à appliquer via la signalisation aux circulations commerciales en cas de fortes rafales de vent.

\subsection{La sécurité et la conception du matériel roulant}

Même si les performances demandées au matériel sont de plus en plus élevées pour des coûts d'achat et de maintenance qu'il convient de bien maîtriser, la sécurité reste la préoccupation première de la phase conception.

Les quelques exemples présentés ci-après ont pour ambition de montrer l'importance de la place des essais dans la conception et la validation des parties mécaniques des matériels roulants.

La défaillance d'une pièce ou d'un organe peut toujours être la cause d'accident corporel voire de circulation, c'est pourquoi ils sont soumis à des référentiels spécifiques stricts en matière tant de conception que de fabrication.

Aussi, les éléments fortement sollicités en dynamique tels que la caisse du véhicule, les roues, les essieux, les freins, les suspensions, les bogies et les pantographes font l'objet d'une attention renforcée suivant le schéma de la figure 3 .

Les moyens de calcul sont de plus en plus performants et permettent d'optimiser les conceptions.

Toutefois, deux paramètres restent déterminants et nécessitent des investigations poussées.

Il s'agit de la détermination des chargements conventionnels représentatifs de la vie d'un matériel et des critères d'acceptation.

C'est pourquoi les chargements conventionnels se doivent d'intégrer les sollicitations dynamiques du service. Ceci est habituellement réalisé par des combinaisons de chargements statiques et par des dépouillements spécifiques de résultats de mesures d'essai.

Les critères d'acceptation des matériels sont élaborés à partir de valeurs provenant des lois de comportement des matériaux, en statique et en fatigue monoaxiale monotone, pondérées par des coefficients de sécurité issus de l'expérience acquise.

D'autres critères permettant une prise en compte plus précise des chargements réels, souvent multiaxiaux et aléatoires, sont à l'étude.

La conception est systématiquement suivie d'une validation.

Les essais entrepris à cette occasion permettent non seulement d'entériner ou non les choix technologiques et les dispositions constructives retenus mais aussi d'enrichir des bases de données qui seront exploitées pour affiner les méthodologies de conception. Pour compléter les essais correspondant aux chargements conventionnels utilisés à la phase calcul, des matrices de chargements réels, provenant de mesures en ligne, sont rejouées sur banc d'essais pour déterminer les lois d'endommagement (Fig. 4).

Jadis, des essais conventionnels statiques sur structures (Fig. 5) suffisaient à qualifier un matériel.

Devant les défaillances rencontrées en service, il s'est révélé indispensable de dimensionner et de tester les structures à la fatigue et de simuler sur banc d'essai trente ans de vie du matériel.

Cette démarche s'applique tout particulièrement aux châssis de bogie (Fig. 6), structures hautement sollicitées en dynamique lors des circulations ferroviaires.

Puis, compte tenu des progrès technologiques, le concept de sécurité passive a fait son apparition à la SNCF [6].

Depuis la mise en service du TGV DUPLEX (2 niveaux), les matériels récents comme les automotrices XTER ou ATER comportent des zones déformables d'absorption d'énergie et des zones de survie indéformables où sont situés les voyageurs et le mécanicien (Fig. 7).

La sécurité passive n'a pas pour vocation de combler d'éventuelles lacunes en matière de sécurité active ${ }^{1}$ mais plutôt de la compléter notamment face aux événements non maîtrisables voire de limiter les dommages en cas

\footnotetext{
${ }^{1}$ Sous ce terme, on désigne les équipements (système de signalisation, contrôle de vitesse, ....) et les procédures qui permettent d'assurer la sécurité des circulations (espacement, rattrapage, collision...).
} 


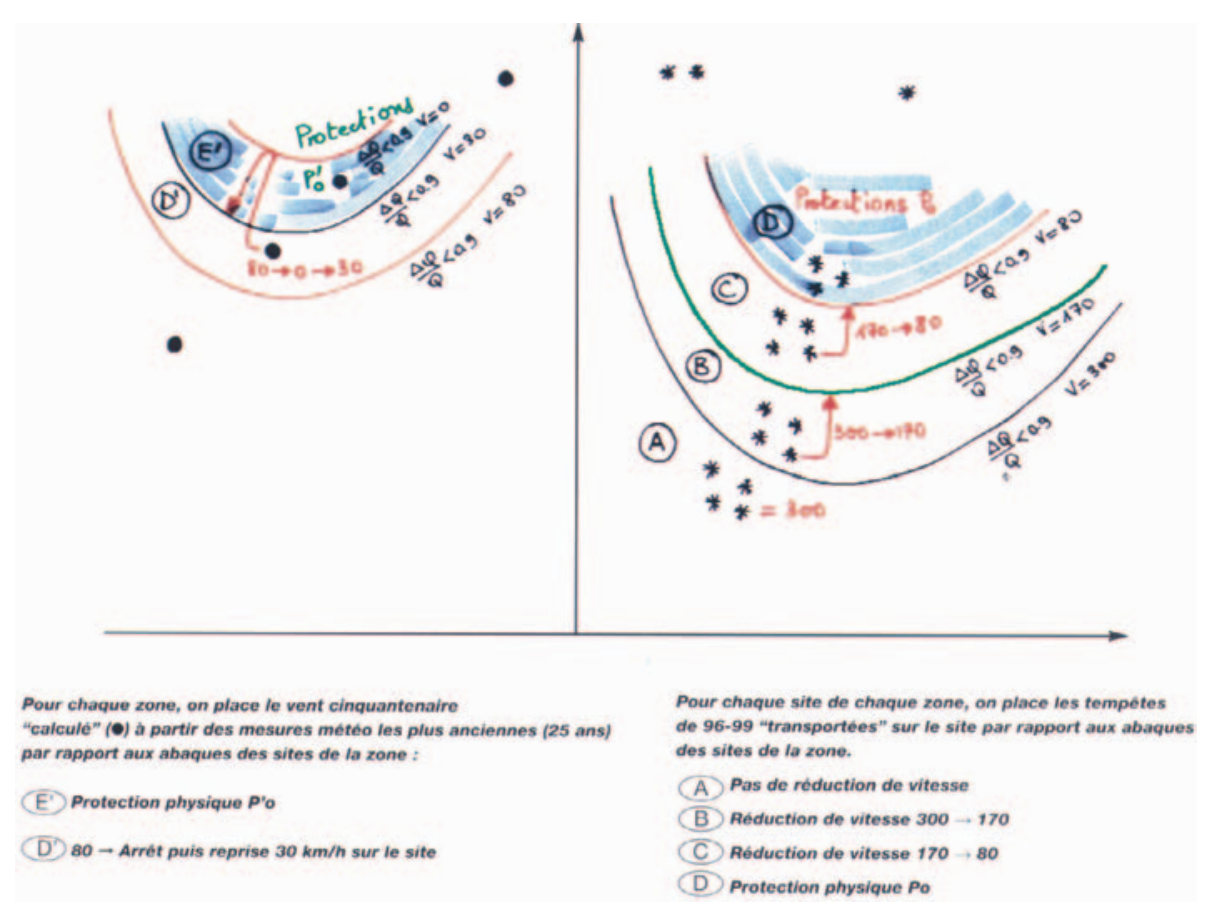

Fig. 2. Schématisation des abaques limites.

de collision. Toutefois, le cahier des charges associé à la sécurité passive se doit de rester réaliste.

En effet, la prise en considération d'un choc frontal de deux TGV roulant à $300 \mathrm{~km} \mathrm{~h}^{-1}$ conditionnerait un nez de motrice d'environ $300 \mathrm{~m}$ de long, capable d'absorber par déformation plastique sous un effort constant de $4500 \mathrm{kN}$ une énergie de 1400 MJ.

L'analyse des accidents survenus lors des dix dernières années a permis de mettre en évidence un accident type de référence. Il s'agit de l'accident au passage à niveau de Voiron (Isère) entre une rame TGV Grenoble-Lyon et un transport exceptionnel de vingt tonnes immobilisé transportant un bloc rigide de soixante tonnes. La vitesse lors du choc était de $110 \mathrm{~km} \mathrm{~h}^{-1}$.

Les nouvelles dispositions constructives mises en œuvre ne correspondent qu'à moins de $1 \%$ du coût du matériel. Les essais réalisés sur des structures à l'échelle une permettent de valider les codes de calcul nécessaires aux simulations.

Le freinage reste l'élément de base de la sécurité.

On admet volontiers de pouvoir être transporté de plus en plus vite sous réserve de pouvoir s'arrêter rapidement en toutes circonstances quelles que soient les conditions extérieures.

Cette gageure passe par la qualification des matériaux de freinage mais aussi par des essais d'endurance et de performance des dispositifs de freinage (Fig. 8).

Lors de ces essais, les couples de frottement sont caractérisés, les distances d'arrêt mesurées et le comportement thermomécanique identifié [7].

Un bas facteur de frottement (ex. fonte) ne permet pas d'excéder des vitesses de circulation de $200 \mathrm{~km} \mathrm{~h}^{-1}$. Le freinage de référence de demain correspondra pour un disque à une énergie de $31 \mathrm{MJ}$ à $380 \mathrm{~km} \mathrm{~h}^{-1}$.
De plus, le freinage est le poste de consommation de pièces de rechange (semelles de freinage, garnitures pour disques, ...) le plus important pour la SNCF après les organes de roulements.

L'ultime et indispensable essai concerne la dynamique ferroviaire où il est vérifié que le matériel ne présente pas de risque de déraillement ou qu'il n'est pas susceptible de faire riper la voie lors des circulations.

Lors de marches d'essais caractéristiques en termes de vitesses, alignements, rayons de courbure, dévers..., des mesures des forces verticales et transversales exercées par les roues sur le rail sont pratiquées parallèlement à des mesures accélérométriques qualifiant le comportement vibratoire (Fig. 9).

Bien sûr, on ne peut pas aborder la sécurité sans parler de la tenue au feu des matériaux utilisés.

Dans ce domaine aussi, d'énormes progrès ont été réalisés et les critères d'acceptation sont draconiens [8].

Il n'est plus acceptable de voir un véhicule totalement incinéré en moins de trente minutes à partir d'un banal petit feu de papier journal dans un compartiment comme ce fut le cas pour l'essai réalisé en 1978.

Aujourd'hui, on recherche l'extinction spontanée.

La sélection des matériaux se fait à l'aide de grilles d'acceptation (Fig. 10) élaborées à partir de l'aptitude d'un matériau à favoriser ou non la combustion (classements $\mathrm{M}$ ou I) et à partir de la toxicité et de l'opacité des fumées émises lors de la combustion (classements F). 


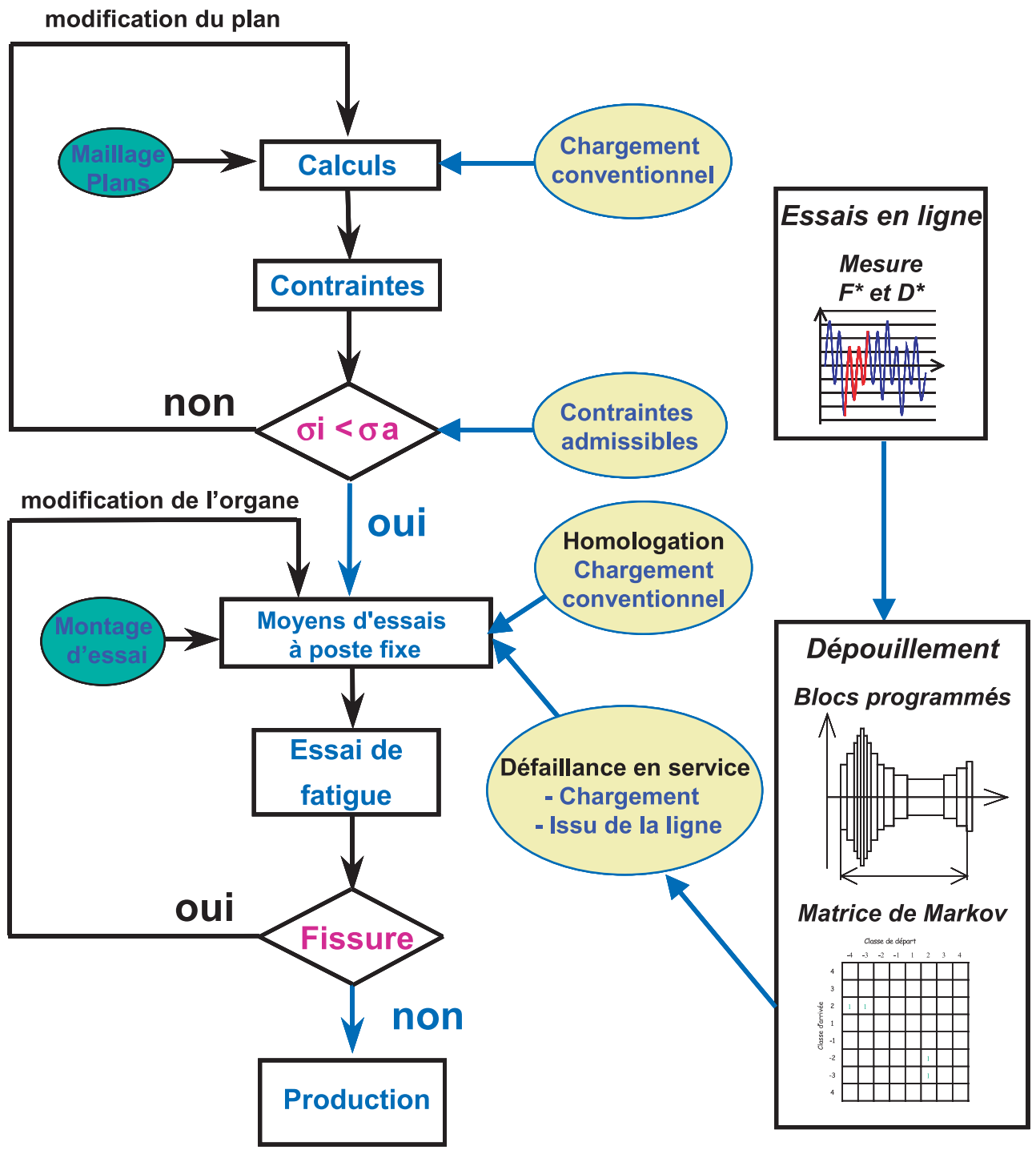

Fig. 3. Logigramme de conception.

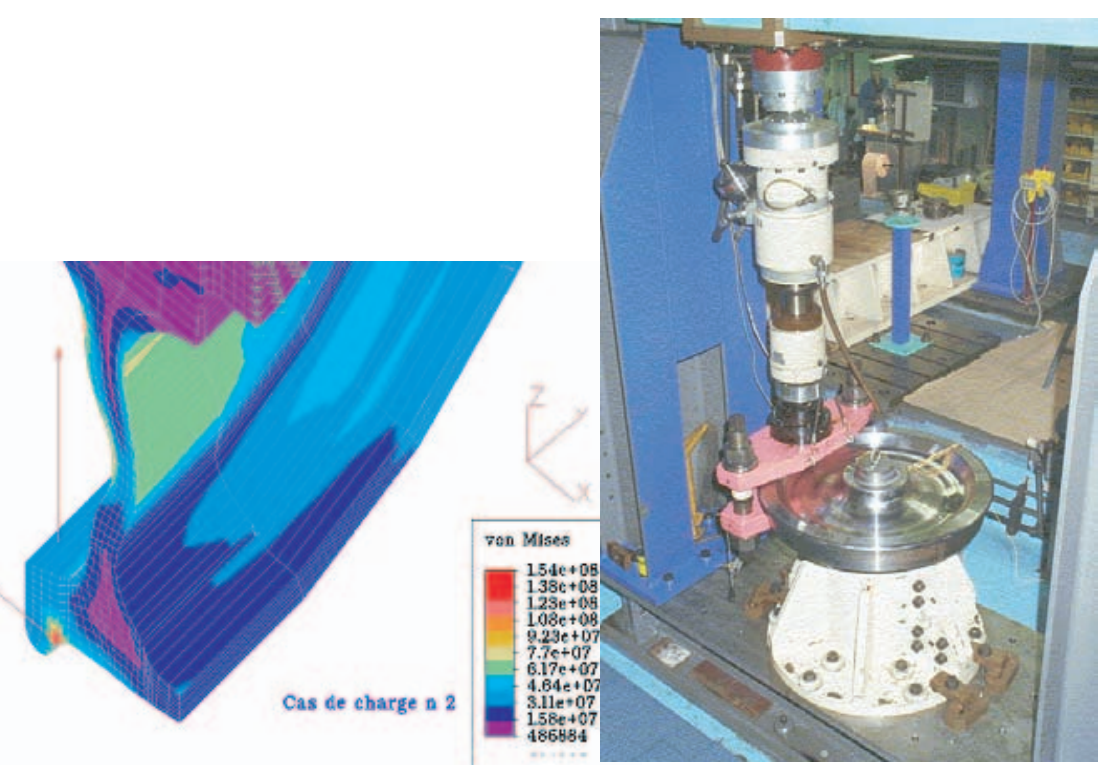

Fig. 4. Conception et essais au banc de fatigue d'une roue monobloc. 


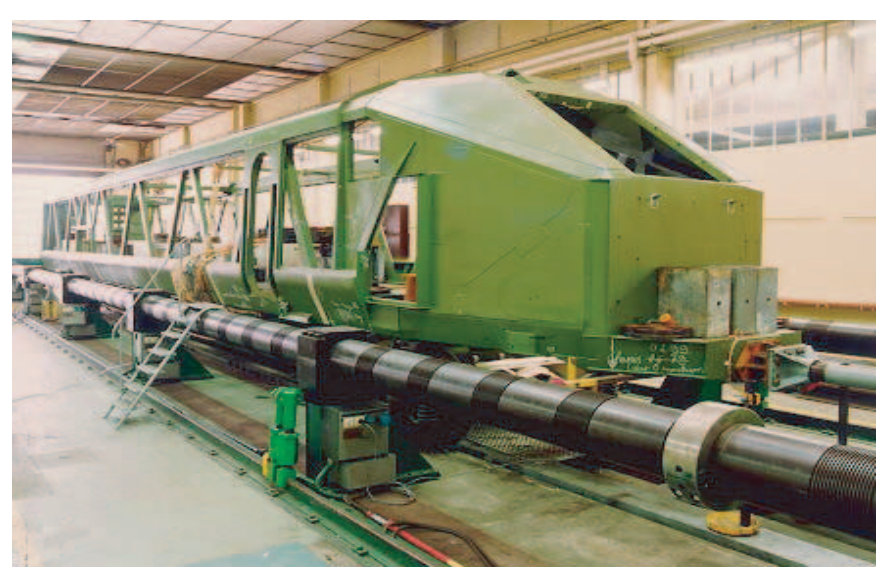

Fig. 5. Essai statiaue sur structure de motrice TGV.

\subsection{La sécurité et la maintenance}

Tout au long de la vie d'un véhicule, il est nécessaire de pratiquer des contrôles non destructifs pour s'assurer de la fiabilité des organes.

Une trame de surveillance évolutive est établie en fonction des sollicitations dynamiques et des modifications éventuelles que subissent les organes en service pendant un temps ou un parcours donné.

Ainsi, les axes des essieux, exposés en service à des facteurs dégradants tels que corrosion sur corps et sur portée de calage, sont surveillés par ultrasons et par magnétoscopie (Fig. 11).

Ils sont soumis au préalable en fabrication à une recherche des défauts internes et d'un éventuel manque de perméabilité ultrasonore.

Des comptes-rendus de contrôle sont établis et enregistrés au numéro de l'essieu. Ces résultats sont analysés dans le cadre d'investigations sur l'évolution des trames de maintenance.

Les méthodes de contrôle sont choisies en fonction des pièces à examiner, de la sensibilité de détection souhaitée et de l'accessibilité. Elles sont validées par un expert niveau 3 COFREND $^{2}$.

Certains centres d'entretien possèdent des installations automatiques pour le contrôle aux ultrasons des roues et des essieux.

L'évolution vers la sûreté des organes de sécurité passe par une amélioration de la sensibilité de détection des défauts qui peuvent apparaître en fabrication et en service.

\section{En matière d'environnement}

\subsection{Le respect de l'environnement}

Moindre consommation d'espace, faibles pollutions atmosphérique et acoustique, émissions de gaz à effet de

\footnotetext{
${ }^{2}$ L'AEF est centre de qualification du comité sectoriel CFCM (Comité Ferroviaire de Certification pour la Maintenance) de la COFREND (Confédération Française pour les essais Non Destructifs).
}

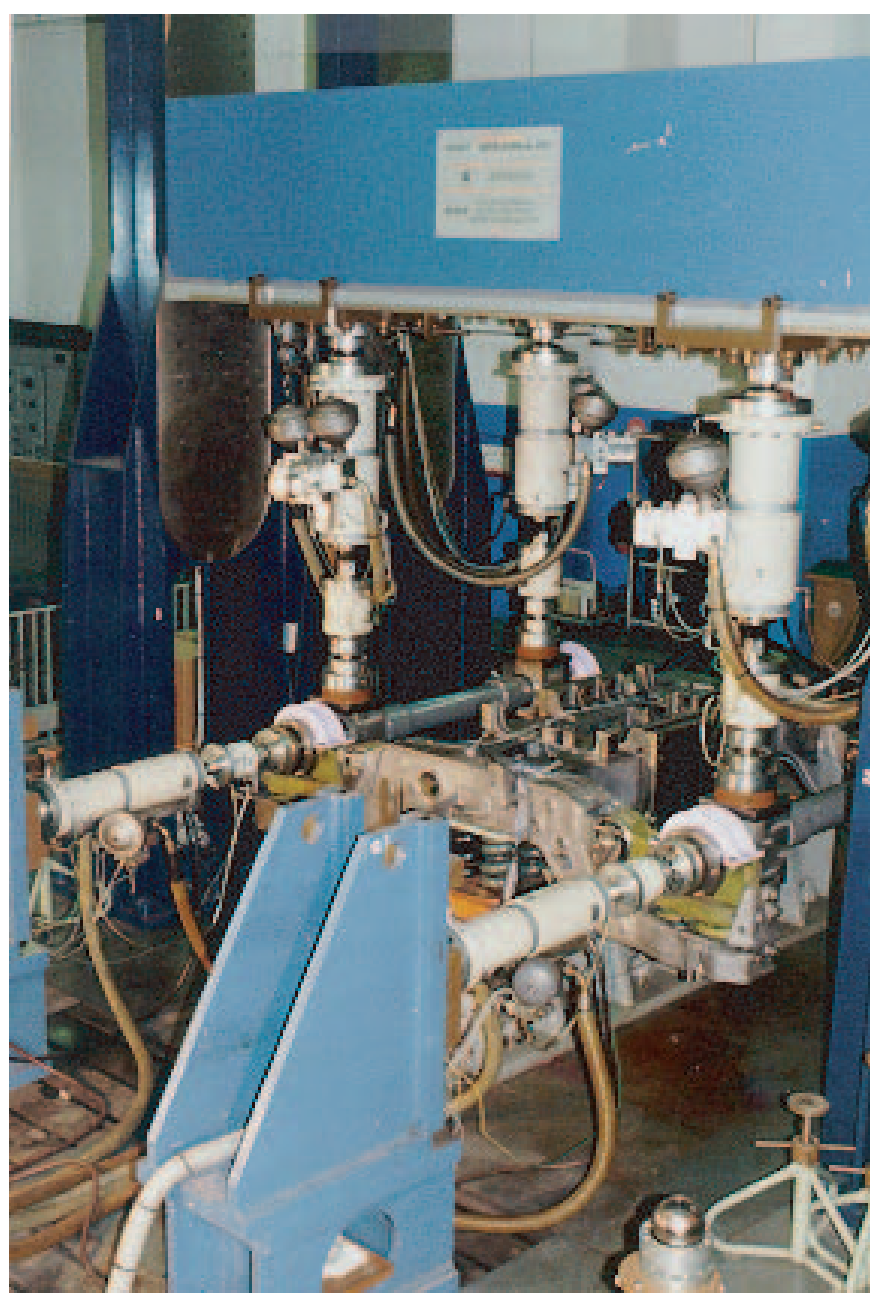

Fig. 6. Châssis de bogie sur banc d'essai de fatigue.

serre relativement limitées, les atouts environnementaux du chemin de fer sont considérables.

En effet, si la largeur moyenne d'une plate-forme TVG est de 15 mètres, il faut prévoir 28 mètres pour une autoroute de deux fois deux voies (Fig. 12).

Concernant le transport aérien, la surface d'emprise totale de l'aéroport Charles-de-Gaulle est de 3000 hectares (un tiers de la surface de Paris); celle de la ligne Grande Vitesse de Paris à Lyon n'est que de 2400 hectares pour un nombre de voyageurs comparable.

La maîtrise de la végétation dans les emprises ferroviaires, en particulier par l'utilisation de produits phytosanitaires de désherbage ou de débroussaillage, s'impose à la SNCF pour des raisons techniques et de sécurité.

La végétation est en effet une cause importante de dégradation de la voie et de la plate-forme. De plus, elle diminue l'efficacité du freinage (dépôt sur les rails, feuilles mortes, ... ) et entrave la visibilité des conducteurs et des personnels de maintenance de l'infrastructure.

Les études d'optimisation menées entre 1984 et 1999 ont permis une diminution de $40 \%$ du tonnage annuel total de matières actives avec un effort considérable ces dernières années. 


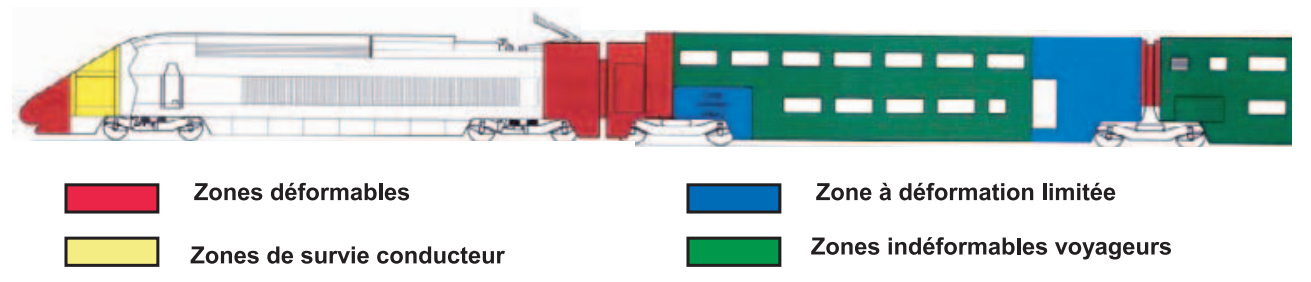

Fig. 7. Dispositions constructives en matière de sécurité passive sur TGV.



Fig. 8. Essai de freinage au banc.

Pour ce faire, la SNCF a mis au point un matériel d'épandage équipé d'un dispositif à mélange continu eauproduits actifs avec un débit proportionnel à la vitesse du train et dont la largeur arrosée est réglable avec une grande précision.

\subsection{La pollution atmosphérique}

La situation de la SNCF, en matière d'émissions polluantes de son parc d'engins Diesel (près de 4000 engins avec une moyenne d'âge de plus de 25 ans), est très contrastée.

Le meilleur côtoie le moins bon; à quelques situations de crise en sites urbains il faut opposer une contribution quasi négligeable de la SNCF aux pollutions

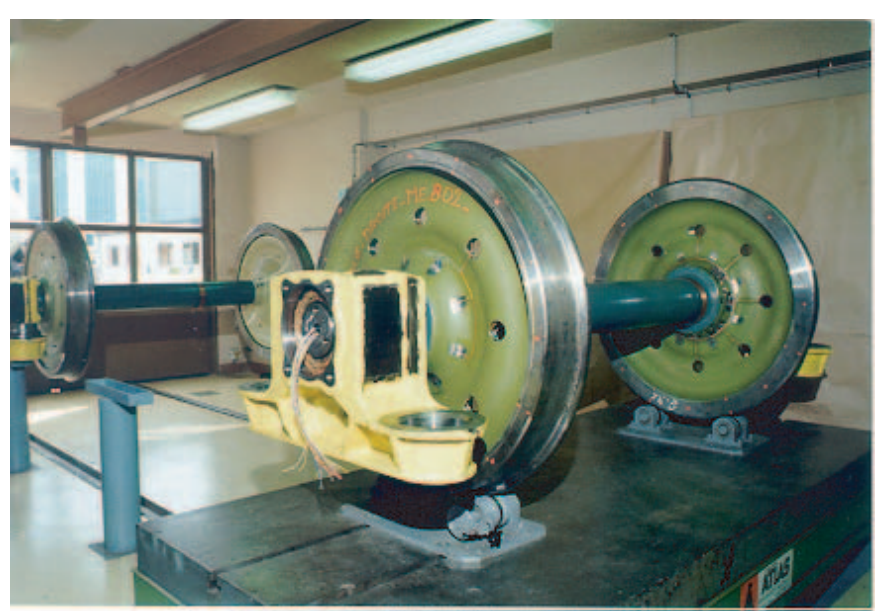

Fig. 9. Essieu de mesure de l'interaction roue-rail.
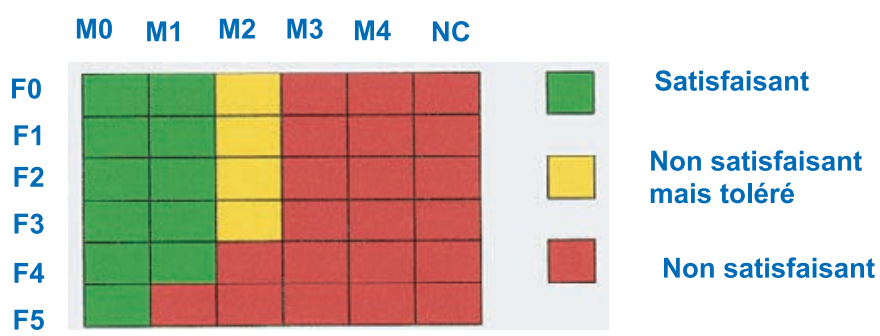

Fig. 10. Exemple de grille d'acception d'un matériau.

atmosphériques à l'échelle globale des transports urbains en France.

Contraste ensuite entre les progrès proposés par les motoristes du secteur routier et la relative lenteur des progrès des secteurs ferroviaire, fluvial et maritime.

Contraste enfin entre l'idée d'un immobilisme que certains diffusent et les plans d'action décidés par l'entreprise.

Ces actions portent notamment sur l'achat de nouveaux engins Diesel équipés de moteurs non polluants, le renouvellement ou la remotorisation d'une partie du parc Diesel, la mise au point de kits de dépollution, le déplacement des lieux de stationnement et de chauffage des engins dans des secteurs moins sensibles, ainsi que sur l'utilisation d'huiles de meilleure qualité et le choix d'un gasoil en basse teneur en soufre.

Actuellement, un système d'aspiration et de traitement des fumées est en expérimentation sur un site de préchauffage et des mesures de qualité de l'air sont régulièrement pratiquées en gare (Fig. 13). 


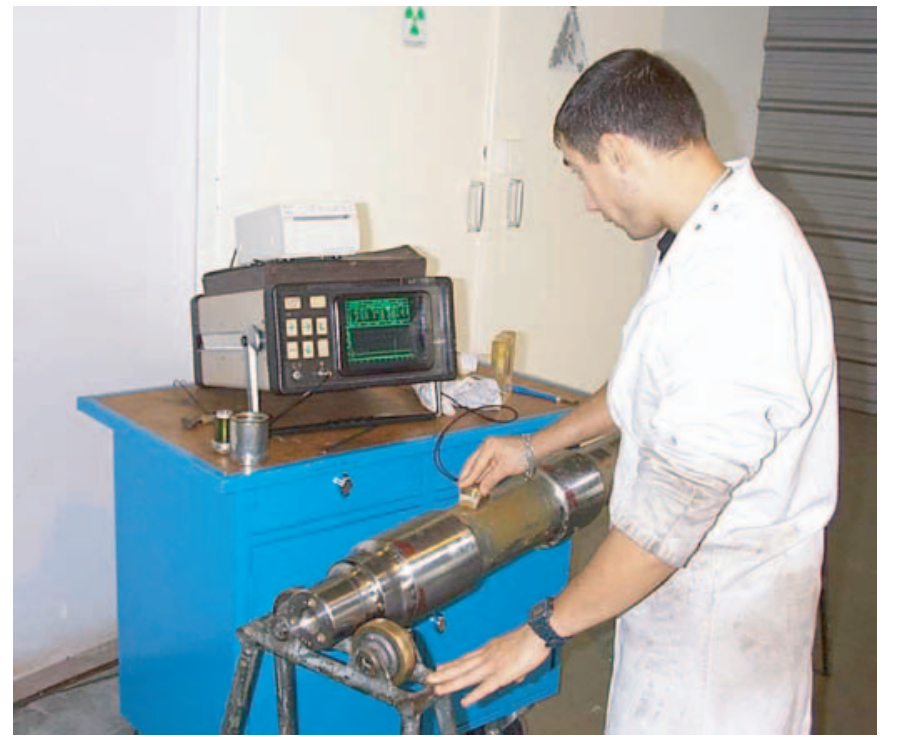

Fig. 11. Opérateur CND sur essieu-axe.



Fig. 12. Emprise ferroviaire à proximité d'une emprise autoroutière.

\subsection{Le bruit}

Le bruit apparaît comme la première nuisance environnementale perçue par les français dans leur vie quotidienne.

Sujet particulièrement sensible, le bruit est un phénomène physique complexe qu'il est nécessaire d'appréhender au mieux pour contribuer à sa réduction.

Le bruit ferroviaire est principalement émis à l'interface entre le matériel roulant et l'infrastructure, au contact roue-rail.

Les signatures bruit des matériels roulants sont très diverses (Fig. 14), le paramètre majeur étant l'état de surface des tables de roulement des roues des véhicules - plus la rugosité est faible, plus le bruit émis est faible. D'autre part, le même véhicule émettra un niveau de bruit très variable en fonction des paramètres de l'infrastructure; un bon état de surface du rail est également primordial.

Enfin, la propagation du bruit est liée à la configuration de l'infrastructure au sein de son environnement

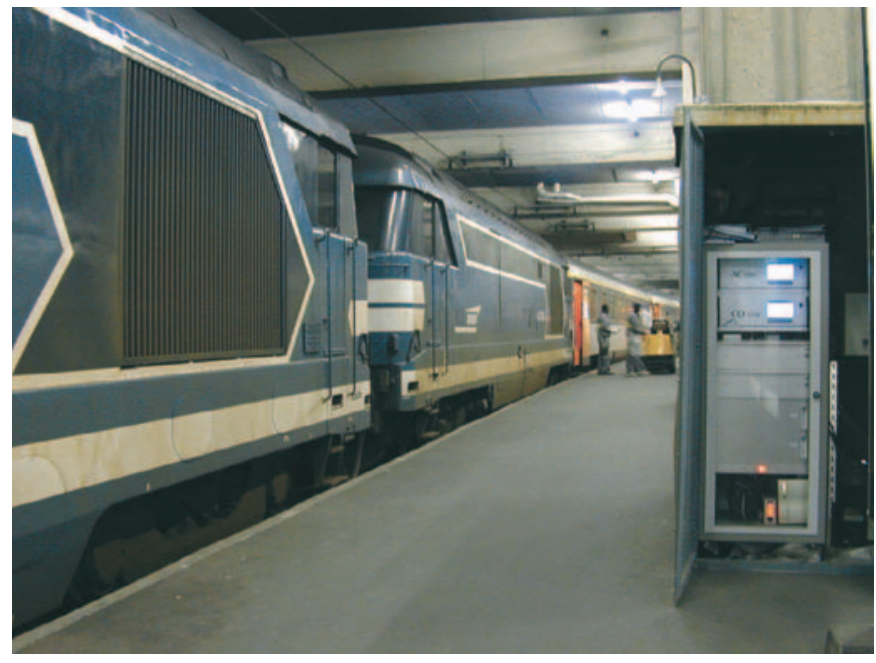

Fig. 13. Qualité de l'air en gare.

(déblai, remblai... ) et le niveau de gêne est bien sûr lié à la proximité de bâtiments d'habitation.

Par conséquent, une approche système est indispensable pour lutter contre les nuisances sonores ferroviaires.

L'action principale à mener pour réduire le niveau de bruit à l'émission des matériels ferroviaires consiste à modifier ou à supprimer les semelles de frein s'appliquant directement sur les roues tout en conservant les performances de freinage.

Ce problème concerne essentiellement les matériels fret avec les 70000 wagons en France et les 700000 susceptibles de s'échanger en Europe pour lesquels il n'a pas été trouvé à ce jour de solution économique de substitution des semelles fonte par un matériau composite moins agressif vis-à-vis des tables de roulement et présentant des caractéristiques de freinage équivalentes.

L'Agence d'Essai Ferroviaire contribue d'une manière importante aux recherches dans ce domaine par les essais tant sur banc qu'en ligne qu'elle met en œuvre.

Pour le fret, des récentes mesures de bruit ont montré l'efficacité des écrans acoustiques placés devant les roues de wagons.

La restauration de l'état de surface des rails s'obtient par meulage au moyen de machines malheureusement bruyantes et dont la vitesse d'avancement est faible. Des absorbeurs de bruit constitués de bandes de caoutchouc collées sur l'âme des rails sont en cours de mise au point.

Enfin, en complément du traitement du bruit à la source, des murs antibruit qui permettent une diminution moyenne de $5 \mathrm{~dB}$, voire la descente du profil en long d'une ligne en « enterrant » celle-ci ou le traitement acoustique des façades des logements des riverains d'une ligne ferroviaire sont autant de solutions efficaces mais dont la mise en œuvre reste fort onéreuse. 

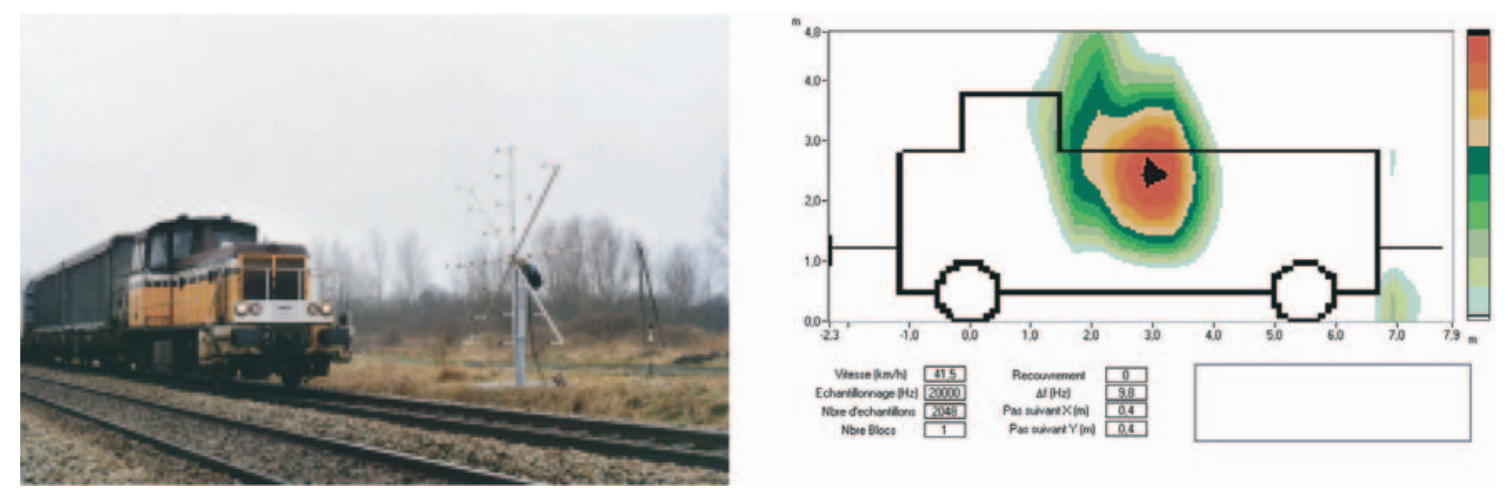

Fig. 14. Signature acoustique d'un locotracteur.

\subsection{Les déchets}

Depuis le $1^{\text {er }}$ juillet 2002, il est interdit en Europe de mettre en décharge tous les déchets recyclables ou valorisables. En outre, le producteur des déchets en est responsable jusqu'à leur élimination finale.

C'est pourquoi les déchets industriels spéciaux (DIS) produits par les activités de l'entreprise ont été répertoriés et font l'objet de fiches définissant les dispositions à prendre en matière d'élimination.

La SNCF produit plus de 40000 tonnes par an de déchets industriels banals (DIB). Aujourd'hui, une part importante de ces déchets tels que les métaux non ferreux ou les déchets d'emballage fait l'objet de tri en vue d'un recyclage afin de respecter la norme ISO 14000.

\section{Conclusion}

À travers ces quelques exemples, nous souhaitons avoir montré l'implication permanente de la SNCF dans le maintien et le développement d'un niveau de sécurité optimal et du développement durable.

La conception et la fabrication des matériels roulants sont dûment testées, validées et contrôlées afin que les dispositions prises correspondent bien aux objectifs visés.
L'insertion environnementale devient un concept majeur pour tout nouveau projet dont les objectifs principaux sont la préservation des milieux naturels et l'insertion phonique.

De même, les circulations quotidiennes sont surveillées, scrutées en permanence et les règles de maintenance sans cesse améliorées.

Si un niveau absolu de sécurité totale n'est malheureusement pas accessible et garantissable, il doit faire l'objet d'une quête permanente et reste une préoccupation essentielle de la SNCF et de l'Agence d'Essai Ferroviaire.

\section{Références}

[1] Au sens de la directive européenne 91/440 du 29 juillet 1991

[2] Loi d'orientation sur les transports intérieurs de 1982 (la «LOTI ») et Loi n 97-135 du 13 février 1997 portant sur la création de $\mathrm{RFF}$

[3] Décret $\mathrm{n}^{\circ}$ 2000-286 du 30 mars 2000

[4] Référentiel Général SNCF RG-0010, Management général de la sécurité des circulations

[5] La sécurité : philosophie et convergences, Colloque AFFI de novembre 2002

[6] Revue Générale des Chemins de fer de novembre 1993

[7] Référentiel UIC 541-3, 4

[8] Norme NF -F 16101 